\title{
Mapping of developmental dysplasia of the hip to two novel regions at 8q23-q24 and 12p12
}

\author{
LIXIN ZHANG ${ }^{*}$, XIAOWEN XU*, YUFAN CHEN, LIANYONG LI, LIJUN ZHANG and QIWEI LI \\ Department of Pediatric Orthopaedics, Shengjing Hospital of China Medical University, \\ Shenyang, Liaoning 110004, P.R. China
}

Received June 15, 2019; Accepted January 1, 2020

DOI: $10.3892 /$ etm.2020.8513

\begin{abstract}
Developmental dysplasia of the hip (DDH), previously known as congenital hip dislocation, is a frequently disabling condition characterized by premature arthritis later in life. Genetic factors play a key role in the aetiology of DDH. In the present study, a genome-wide linkage scan with the Affymetrix 10K GeneChip was performed on a four-generation Chinese family, which included 19 healthy members and 5 patients. Parametric and non-parametric multipoint linkage analyses were carried out with Genespring GT v.2.0 software, and the logarithm of odds (LOD) score and nonparametric linkage (NPL) score were calculated. Parametric linkage analysis was performed, assuming an autosomal recessive trait with full penetrance and Affymetrix 'Asian' allele frequencies. The strongest evidence for linkage was found on chromosome 8q23-24, with a peak LOD score of $2.658(\theta=0)$, covering $2.377 \mathrm{Mb}$ from single nucleotide polymorphisms (SNPs) rs724717 to rs720132. This interval included nine additional successive SNPs: rs1566071, rs1902121, rs756404, rs702768, rs777813, rs2033995, rs147959, rs2884367 and rs1898287. The same region also yielded the highest NPL score of $2.883(\mathrm{P}=0.0156)$ from the non-parametric multipoint linkage analysis. Additionally, the second highest NPL score of $2.727(\mathrm{P}=0.0156)$ and LOD score of $2.528(\theta=0)$ were obtained on chromosome 12p12 for three consecutive markers (rs1919980, rs763853 and rs725124). This region overlapped a narrow distance of $0.642 \mathrm{Mb}$. Notably, in addition to these two regions; no significant linkage was identified for other chromosomal regions (with LOD and NPL scores $>2.0$ ). For the first time, at least for this pedigree, the evidence in the present
\end{abstract}

Correspondence to: Professor Lianyong Li, Department of Pediatric Orthopaedics, Shengjing Hospital of China Medical University, 36 Sanhao Street, Heping, Shenyang, Liaoning 110004, P.R. China

E-mail: loyo_ldy@163.com

${ }^{*}$ Contributed equally

Key words: developmental dysplasia of the hip, gene mapping, linkage, single nucleotide polymorphism, microarray chip study showed that DDH is mapped to two novel regions at 8q23-q24 and 12p12.

\section{Introduction}

Developmental dysplasia of the hip (DDH), previously known as congenital hip dislocation, is a frequently disabling condition characterized by premature arthritis later in life (1). The term encompasses a spectrum of diseases ranging from minor acetabular dysplasia to irreducible dislocation, which affects 25-50 in 1,000 live births among Lapps and Native Americans but is very rare among southern Chinese and African populations (1). A number of factors, including genetic mutations and intrauterine and postnatal environmental factors, are thought to contribute to the disease (2).

Genetic influence on DDH has been long known but is still poorly understood. Although most clinical cases appear to be sporadic, $12-33 \%$ of patients have a family history of DDH $(3,4)$. One study reported a 10 -fold increase in the incidence of DDH among the parents of index patients and a seven-fold increase among the siblings compared with the general population (3), which suggested a complex aetiology involving multiple genes interacting with environmental factors. Several genes, such as collagen alpla-1 (I) chain, homeobox protein Hox-B, basement membrane-specific heparan sulfate proteoglycan core protein, plasma membrane calcium-transporting ATPase 4, pregnancy-associated plasma protein-A2 (PAPPA2), teneurin-3 (TENM3), growth/differentiation factor 5 (GDF5) and transforming growth factor beta-1 proprotein have been investigated in various populations as candidates (5-11). However, to the best of our knowledge, few results have been replicated in other populations, suggesting that geographic and ethnic factors probably also play a key role in disease aetiology. Therefore, locating and subsequently identifying the causative genes for DDH are essential. Two identified genes have been found based on the genetic analysis of large segregating $\mathrm{DDH}$ families after mapping of the causative regions. A variant in the $\mathrm{CX} 3 \mathrm{C}$ chemokine receptor $1(C X 3 C R 1)$ gene was found in all DDH-affected members of a 72-member, four-generation affected family (12). Watson et al (13) identified a mutation in the UFM1 specific peptidase 2 (UFSP2) gene in a family with Beukes hip dysplasia. Genetic variations in other diseases were also found after locating causative regions. Shrimpton et al (14) found 
that chromosome $2 \mathrm{q} 31$ was associated with congenital vertical talus, which led to the detection of a mutation in the homeobox protein Hox-D10 gene using whole-genome linkage analysis. Additionally, the vitamin D receptor gene was associated with late-onset Alzheimer's disease after mapping susceptibility loci to chromosome 12q13 (15).

In the present study, a genome-wide linkage scan with the Affymetrix $10 \mathrm{~K}$ GeneChip was performed on a four-generation Chinese family, which included 19 healthy members and 5 patients affected with DDH. For the first time, at least for this pedigree, the evidence showed that the disease is mapped to two novel regions at 8q23-q24 and 12p12.

\section{Materials and methods}

Patients. The proband (III-6), a 28-year-old female, appeared at the Department of Paediatric Orthopaedics, Shengjing Hospital of China Medical University for consultation for her two-year-old son (IV-3), as she had a family history of DDH. The pedigree of the Chinese family is shown in Fig. 1. The four-generation Chinese family, including 24 individuals, resides in a village in northeast China. Family members were invited to participate in the study, and those aged $\geq 18$ years gave informed consent. Approval was obtained from the Human Research Ethics Committee of Shengjing Hospital of China Medical University. The status of the 24 individuals was established on the basis of clinical and radiological examinations. Five individuals (aged 23-72 years) had DDH, including two males (III-9 and III-10) and three females (I-1, III-6 and III-7). Anterior-posterior radiography of the pelvis revealed that all affected members had similar bilateral hip dislocation, which was classified as grade IV according to the Tönnis classification system (Fig. 2) (16). These individuals did not have any other system abnormalities associated with Down syndrome, Marfan syndrome, Ehlers-Danlos syndrome or dyschondroplasia and were therefore diagnosed with isolated DDH. All other members of the pedigree had normal appearance and height $(163-175 \mathrm{~cm})$.

Genome-wide scan and linkage analysis. Blood samples were obtained from all members of the family except for two individuals (III-3 and III-5). DNA was extracted using QIAamp DNA Blood mini kit (Qiagen $\mathrm{GmbH}$ ) through a standard procedure. A genome-wide scan was performed on the 22 individuals using Affymetrix 10K single nucleotide polymorphism (SNP) arrays at GeneTech Biotechnology Co., Ltd. Parametric and nonparametric multipoint linkage analyses were performed using Genespring GT v.2.0 software (Agilent Technologies, Inc.), and the logarithm of odds (LOD) and NPL scores were calculated (Fig. 3). Parametric linkage analysis was performed, assuming an autosomal recessive trait with full penetrance and Affymetrix 'Asian' allele frequencies.

\section{Results}

The strongest evidence for linkage was found on chromosome 8q23-24, with a peak LOD score of $2.658(\theta=0)$, covering 2.377 $\mathrm{Mb}$ from SNP rs724717 to rs720132. This interval included nine additional successive SNPs: rs1566071, rs1902121, rs756404, rs702768, rs777813, rs2033995, rs147959, rs2884367 and rs1898287. The same region also yielded the highest NPL score of $2.883(\mathrm{P}=0.0156)$ from non-parametric multipoint linkage analysis. Additionally, the second highest NPL score of $2.727(\mathrm{P}=0.0156)$ and an LOD score of $2.528(\theta=0)$ were obtained on chromosome 12p12 for three consecutive markers (rs1919980, rs763853 and rs725124; Table I). This region overlapped a narrow distance of $0.642 \mathrm{Mb}$. Notably, in addition to the two regions, no significant linkage was identified for other chromosomal regions (with both LOD and NPL scores >2.0).

\section{Discussion}

The aetiology of DDH may involve complex interactions between many genes and the environment. Carter and Wilkinson (17) postulated the existence of two genetic systems underlying the disease: The former, polygenic, is related to dysplasia of the acetabulum, and the latter, possibly dominant, controls the capsule around the hip joint. In accordance with the above hypothesis, Wynne-Davies (18) proposed that two aetiologic subtypes of DDH may be observed: One with acetabular dysplasia and another with joint laxity. During the past decade, genome-wide scans and association studies of large families have mapped DDH to chromosome regions $3 \mathrm{p} 22$ (American), 4q35 (Africans), 16p (Icelandic), 13q22 (Japanese), $20 q 11$ (Chinese) and 15q13 and 19p13 (Saudi). Genetic mutations, such as CX3CR1, UFSP2, ubiquinol-cytochrome-c reductase complex, PAPPA2, GDF5 and TENM3, were identified in these regions. The present results and previously identified chromosome regions may be related to the growing development of the hip joint according to the function of genes included in these regions $(10,12,13,19-22)$. DDH was previously associated with the D17S1820 marker from chromosome region 17q21 by analysing 303 individuals from 101 Chinese trios (data not shown). The results were further confirmed by Feldman et al (23) through analysis of a multi-generation American family with 18 members by linkage.

The Chinese family, as reported here, features a rare example of non-syndromic DDH suggestive of an uncommon autosomal recessive model, which is different from previously reported autosomal dominant traits $(4,12)$. However, two unaffected members of the family (III-3 and III-5) refused to provide blood samples, which has limited the power of linkage analysis. Nevertheless, this large pedigree may still be useful for genetic linkage studies to locate candidate genes. For the family, genome-wide scanning has provided strong evidence for regions linked with the disease. Although neither LOD nor NPL scores exceeded 3.0, this may provide a basis for the accumulation of more evidence for the linkage.

Maximum LOD and NPL scores (2.658 and 2.883, respectively) were obtained on chromosome 8q23-24. The region spans $2.377 \mathrm{Mb}$ from SNPs rs724717 to rs720132. Four genes are known to reside in this region, including transcriptional repressor GATA binding 1 (TRPS1), eukaryotic translation initiation factor 3 subunit $\mathrm{H}(E I F 3 H)$, RAD21 cohesin complex component $(R A D 21)$ and rRNA-binding ribosome biosynthesis protein UTP23 (UTP23). Among the four candidates, UTP23 encodes a small subunit processome component, which is required for pre-18S rRNA maturation (24). The product of $R A D 21$ is involved in the repair of double-strand DNA breaks and chromatid cohesion during mitosis (25). Eukaryotic 
Table I. LOD score of two novel chromosome regions.

\begin{tabular}{lcccccccr}
\hline & & \multicolumn{5}{c}{ LOD at $\theta$} \\
\cline { 5 - 9 } Chromosome region & Length $(\mathrm{Mb})$ & Variation & 0 & 0.05 & 0.1 & 0.2 & 0.3 & 0.4 \\
\hline $8 \mathrm{q} 23-\mathrm{q} 24$ & 2.377 & $1-9$ & 2.658 & 2.361 & 2.058 & 1.442 & 0.851 & 0.349 \\
$12 \mathrm{p} 12$ & 0.642 & $10-12$ & 2.528 & 2.240 & 1.946 & 1.349 & 0.777 & 0.297 \\
\hline
\end{tabular}

Numbers (1-9) refer to rs1566071, rs1902121, rs756404, rs702768, rs777813, rs2033995, rs147959, rs2884367 and rs1898287, respectively. Numbers (10-12) refer to rs 1919980, rs763853 and rs725124, respectively. LOD, logarithm of odds.

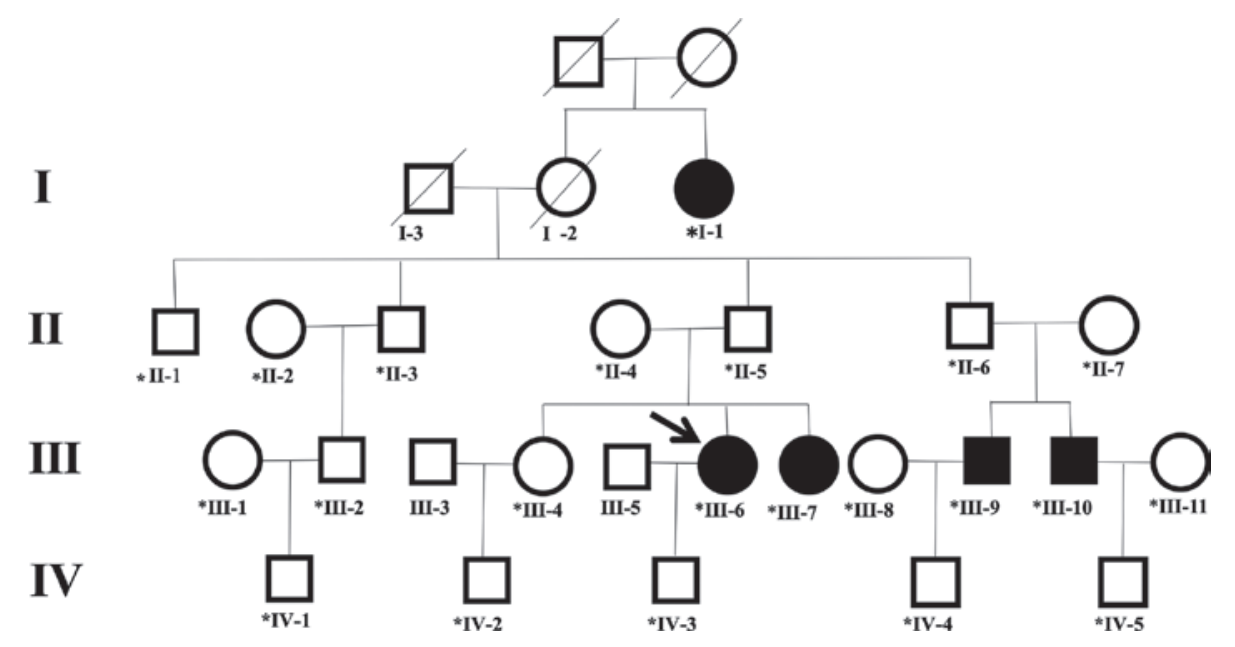

Figure 1. Pedigree of the four-generation Chinese family affected with dysplasia of the hip. The arrow indicates the proband. Asterisks indicate family members whose samples were used for the genome-wide scan.
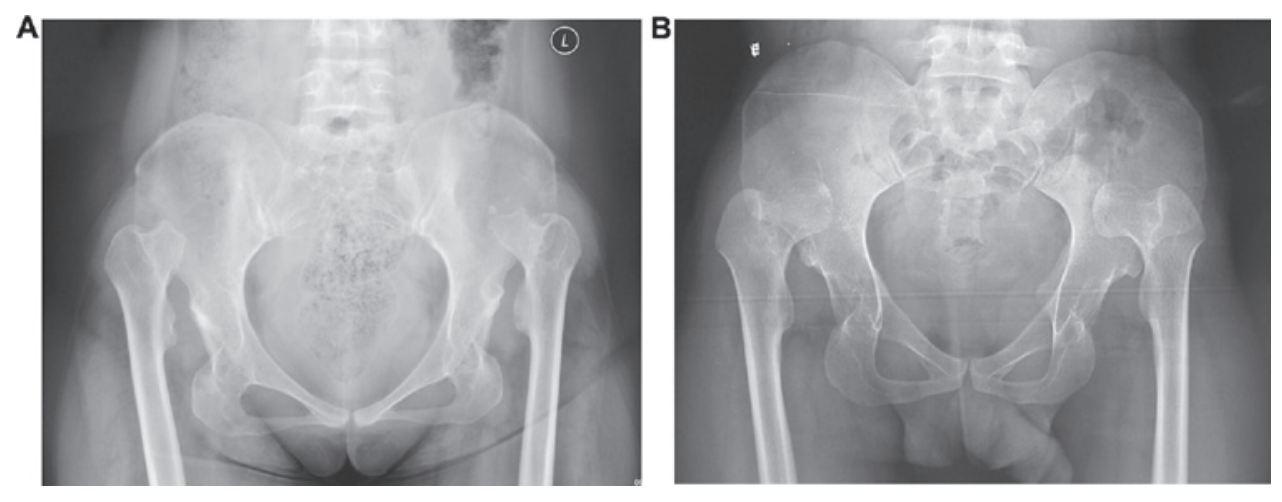

Figure 2. Radiographs showing a Tönnis grade IV bilateral hip dislocation of (A) proband III-6 and (B) proband III-9.

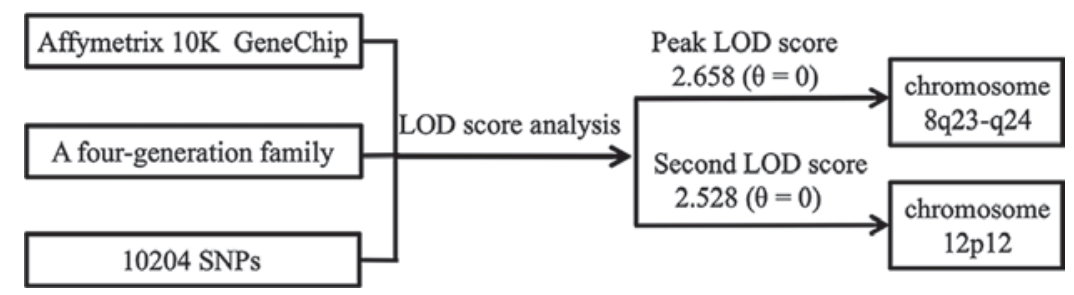

Figure 3. Nucleotide polymorphism array analysis workflow chart. SNP, single nucleotide polymorphism; LOD, logarithm of odds.

translation initiation factor 3 subunit $\mathrm{H}(E I F 3 H)$ is a subunit of the eukaryotic translation initiation factor 3 complex. The intact $E I F 3 H$ protein contributes to efficient translation initiation on 5 ' leader sequences harbouring multiple upstream open 
reading frames, and normal levels of EIF3H are implicated in growth control through protein synthesis $(26,27)$.

TRPS1 seems to be the most attractive candidate from the 8q23-24 region. TRPS1 binds DNA through a single GATA-type zinc-finger domain that recognizes a consensus DNA sequence. Mutations in the TRPS1 gene may cause tricho-rhino-phalangeal syndrome (TRPS) (28), an autosomal dominant type craniofacial and skeletal dysplasia. In addition to craniofacial deformities, patients with TRPS generally have skeletal anomalies, including short stature, hip abnormalities (dysplasia, dislocation of the hip or joint laxity), cone-shaped epiphyses and premature closure of growth plates, which have reflected defects in endochondral ossification. Long bones of the vertebrate appendicular skeleton are formed through the process of endochondral ossification when initial cartilaginous anlagen are replaced by bone (29). Various transcription factors and signalling pathways may be involved in the regulation of endochondral bone formation. The Runx 2 transcription factor is the master regulator of osteoblast differentiation and is required for chondrocyte hypertrophy. TRPS1 physically interacts with Runx2 and represses Runx2-mediated trans-activation. Transgenic mice experiments demonstrated that loss of repression of the Runx2-Ihh-positive regulatory loop induced by TRPS1 mutation can result in altered endochondral bone formation, which is characterized by the dysregulation of chondrocyte differentiation and uncoupling of processes of perichondrial mineralization and chondrocyte maturation (30). Thus, TRPS1 is the most promising gene and should be investigated in future studies.

SOX5 is the only gene known to reside in the $12 \mathrm{p} 12$ region. The gene encodes a member of the SOX (SRY-related HMG-box) family of transcription factors involved in the regulation of embryonic development and cell fate determination. Sox5, together with Sox6, is essential for the establishment of cartilage growth plates and thus the proper and timely development of endochondral bones through regulation of chondrocyte differentiation and proliferation (31). Foetuses of Sox5-Sox6 double null mice may die with severe, generalized chondrodysplasia and fail to form the epiphyseal plates and endochondral bones. Notably, chondrodysplasia can also be observed at the dysplastic acetabulum in DDH, albeit mild and partial. SOX5 gene knockdown inhibited MMP-9 gene expression, contributing to fibroblast survival, proliferation, migration and invasion in a rheumatoid arthritis study (32). Therefore, the potential effects of the SOX 5 gene on the development of non-syndrome DDH cannot be excluded.

According to the analysis above, the two novel regions may affect articular cartilage formation, not only through regulating chondrocyte differentiation and proliferation, but also by uncoupling processes of perichondrial mineralization and chondrocyte maturation. They may also be related to joint development by promoting fibroblast growth.

As a complex disease, $\mathrm{DDH}$ is genetically heterogeneous due to population differences and the complexity of hip development. It is likely that different candidate genes have contributed to the susceptibility to this disorder in different populations. The present study showed a genome-wide linkage analysis for a Chinese DDH family and the identification of two novel loci at 8q23-24 and 12p12. The present results indicated the presence of a disease-associated mutation or polymorphism in the mapped regions of the affected members. With a number of candidate genes identified from the two regions (TRPSI and SOX5 in particular), mutations in these genes can be screened through sequence analysis. These studies may lead to the eventual identification of novel mutations or polymorphisms predisposing individuals to the disease, which may enable early diagnosis and optimal treatment.

To conclude, the evidence presented showed that developmental dysplasia of the hip is mapped to two novel regions at $8 \mathrm{q} 23-\mathrm{q} 24$ and $12 \mathrm{p} 12$. The present results indicate the presence of a disease-associated mutation or polymorphism in the mapped regions for the affected members.

\section{Acknowledgements}

The authors would like to thank Professor Lili Wang (Key Laboratory of the Health Ministry for Congenital Malformation, Shengjing Hospital of China Medical University) for her help in the preparation of the manuscript.

\section{Funding}

The study was supported by the National Natural Science Foundation of China (grant no, 81772296).

\section{Availability of data and materials}

The datasets used and/or analysed during the current study are available from the corresponding author on reasonable request.

\section{Authors' contributions}

LL contributed to the conception of the study. YC and QL contributed significantly to data analysis and manuscript preparation. XX and LXZ analysed the data and wrote the manuscript. LJZ helped perform data analysis with constructive discussions. All authors read and approved the final manuscript.

\section{Ethics approval and consent to participate}

Ethics approval was obtained from the Human Research Ethics Committee of Shengjing Hospital of China Medical University. Family members agreed to be included in this research study.

\section{Patient consent for publication}

Consent for publication was obtained from participants.

\section{Competing interests}

The authors declare that they have no competing interests.

\section{References}

1. Kotlarsky P, Haber R, Bialik V and Eidelman M: Developmental dysplasia of the hip: What has changed in the last 20 years? World J Orthop 6: 886-901, 2015. 
2. Stevenson DA, Mineau G, Kerber RA, Viskochil DH, Schaefer C and Roach JW: Familial predisposition to developmental dysplasia of the hip. J Pediatr Orthop 29: 463-466, 2009

3. Benson MKD: Developmental dysplasia of the hip: Early diagnosis and management. Curr Paediatr 6: 2-8, 1996.

4. Ceylaner G, Ceylaner S, Ustünkan F and Inan M: Autosomal dominant inheritance of congenital dislocation of the hip in 16 members of a family. Acta Orthop Traumatol Turc 42: 289-291, 2008 (In Turkish).

5. Zhao L, Tian W, Pan H, Zhu X, Wang J, Cheng Z, Cheng L, Ma X and Wang B: Variations of the COL1A1 gene promoter and the relation to developmental dysplasia of the hip. Genet Test Mol Biomarkers 17: 840-843, 2013.

6. Wang K, Shi D, Zhu P, Dai J, Zhu L, Zhu H, Lv Y, Zhao B and Jiang Q: Association of a single nucleotide polymorphism in Tbx4 with developmental dysplasia of the hip: A case-control study. Osteoarthritis Cartilage 18: 1592-1595, 2010

7. Jia J,LiL,Zhao Q,Zhang L, Ru J,Liu X,LiQ and ShiL: Association of a single nucleotide polymorphism in pregnancy-associated plasma protein-A2 with developmental dysplasia of the hip: A case-control study. Osteoarthritis Cartilage 20: 60-63, 2012.

8. Zhao L, Pan H, Wang J, Cheng Z, Cheng L, Wang B and Ma X: Two single nucleotide polymorphisms in the GDF5 gene are associated with development dysplasia of the hip in Chinese female population. Sci China Life Sci 56: 1063-1065, 2013.

9. Kolundžić R, Trkulja V, Mikolaučić M, Kolundžić MJ, Pavelić SK and Pavelić K: Association of interleukin-6 and transforming growth factor- $\beta 1$ gene polymorphisms with developmental hip dysplasia and severe adult hip osteoarthritis: A preliminary study. Cytokine 54: 125-128, 2011

10. Basit S, Albalawi AM, Alharby E and Khoshhal KI: Exome sequencing identified rare variants in genes HSPG2 and ATP2B4 in a family segregating developmental dysplasia of the hip. BMC Med Genet 18: 34, 2017.

11. Feldman G, Kappes D, Mookerjee-Basu J, Freeman T, Fertala A and Parvizi J: Novel mutation in Teneurin 3 found to co-segregate in all affecteds in a multi-generation family with developmental dysplasia of the hip. J Orthop Res 37: 171-180, 2019.

12. Feldman GJ, Parvizi J, Levenstien M, Scott K, Erickson JA, Fortina P, Devoto M and Peters CL: Developmental dysplasia of the hip: Linkage mapping and whole exome sequencing identify a shared variant in CX3CR1 in all affected members of a large multigeneration family. J Bone Miner Res 28: 2540-2549, 2013

13. Watson CM, Crinnion LA, Gleghorn L, Newman WG, Ramesar R, Beighton P and Wallis GA: Identification of a mutation in the ubiquitin-fold modifier 1-specific peptidase 2 gene, UFSP2, in an extended South African family with Beukes hip dysplasia. S Afr Med J 105: 558-563, 2015.

14. Shrimpton AE, Levinsohn EM, Yozawitz JM, Packard DS Jr, Cady RB, Middleton FA, Persico AM and Hootnick DR: A HOX gene mutation in a family with isolated congenital vertical talus and Charcot-Marie-Tooth disease. Am J Hum Genet 75: 92-96, 2004.

15. Wang L, Hara K, Van Baaren JM, Price JC, Beecham GW, Gallins PJ, Whitehead PL, Wang G, Lu C, Slifer MA, et al: Vitamin D receptor and Alzheimer's disease: A genetic and functional study. Neurobiol Aging 33: 1844.e1-9, 2012.

16. Bolland BJ, Wahed A, Al-Hallao S, Culliford DJ and Clarke NM: Late reduction incongenital dislocation of the hip and the need for secondary surgery: Radiologic predictors and confounding variables. J Pediatr Orthop 30: 676-682, 2010.

17. Carter $\mathrm{C}$ and Wilkinson J: Persistent joint laxity and congenital dislocation of the hip. J Bone Joint Surg Br 46: 40-45, 1964.
18. Wynne-Davies R: A family study of neonatal and late-diagnosis congenital dislocation of the hip. J Med Genet 7: 315-333, 1970.

19. Sun Y, Wang C, Hao Z, Dai J, Chen D, Xu Z, Shi D, Mao P, Teng H, Gao X, et al: A common variant of ubiquinol-cytochrome c reductase complex is associated with DDH. PLoS One 10: e0120212, 2015.

20. Ingvarsson T, Stefánsson SE, Gulcher JR, Jónsson HH, Jónsson H, Frigge ML, Pálsdóttir E, Olafsdóttir G, Jónsdóttir T, Walters GB, Lohmander LS and Stefánsson K: A large Icelandic family with early osteoarthritis of the hip associated with a susceptibility locus on chromosome 16p. Arthritis Rheum 44: 2548-2555, 2001.

21. Zamborsky R, Kokavec M, Harsanyi S, Attia D and Danisovic L: Developmental dysplasia of hip: Perspectives in genetic screening. Med Sci (Basel) 7: pii: E59, 2019.

22. Basit S, Alharby E, Albalawi AM and Khoshhal KI: Whole genome SNP genotyping in a family segregating developmental dysplasia of the hip detected runs of homozygosity on chromosomes $15 \mathrm{q} 13.3$ and 19p13.2. Congenit Anom (Kyoto) 58: 56-61, 2018.

23. Feldman G, Dalsey C, Fertala K, Azimi D, Fortina P, Devoto M, Pacifici M and Parvizi J: The Otto Aufranc Award: Identification of a $4 \mathrm{Mb}$ region on chromosome $17 \mathrm{q} 21$ linked to developmental dysplasia of the hip in one 18-member, multigeneration family. Clin Orthop Relat Res 468: 337-344, 2010.

24. Lu J, Sun M and Ye K: Structural and functional analysis of Utp23, a yeast ribosome synthesis factor with degenerate PIN domain. RNA 19: 1815-1824, 2013.

25. Beauchene NA, Díaz-Martínez LA, Furniss K, Hsu WS, Tsai HJ, Chamberlain C, Esponda P, Giménez-Abián JF and Clarke DJ: $\operatorname{Rad} 21$ is required for centrosome integrity in human cells independently of its role in chromosome cohesion. Cell Cycle 9: 1774-1780, 2010

26. Choudhuri A, Maitra U and Evans T: Translation initiation factor eIF3h targets specific transcripts to polysomes during embryogenesis. Proc Natl Acad Sci USA 110: 9818-9823, 2013.

27. Hronová V, Mohammad MP, Wagner S, Pánek J, Gunišová S, Zeman J, Poncová K and Valášek LS: Does eIF3 promote reinitiation after translation of short upstream ORFs also in mammalian cells? RNA Biol 14: 1660-1667, 2017.

28. Smaili W, Elalaoui SC, Meier S, Zerkaoui M, Sefiani A and Heinimann K: A novel TRPS1 mutation in a Moroccan family with Tricho-rhino-phalangeal syndrome type III: Case report. BMC Med Genet 18: 50, 2017.

29. Lui JC, Nilsson O and Baron J: Recent research on the growth plate: Recent insights into the regulation of the growth plate. J Mol Endocrinol 53: T1-T9, 2014

30. Zhang Y, Xie RL, Gordon J, LeBlanc K, Stein JL, Lian JB, van Wijnen AJ and Stein GS: Control of mesenchymal lineage progression by microRNAs targeting skeletal gene regulators Trps1 and Runx2. J Biol Chem 287: 21926-21935, 2012.

31. Liu CF and Lefebvre V: The transcription factors SOX9 and SOX5/SOX6 cooperate genome-wide through super-enhancers to drive chondrogenesis. Nucleic Acids Res 43: 8183-8203, 2015.

32. Shi Y, Wu Q, Xuan W, Feng X, Wang F, Tsao BP, Zhang M and Tan W: Transcription factor SOX 5 promotes the migration and invasion of fibroblast-like synoviocytes in part by regulating MMP-9 expression in collagen-induced arthritis. Front Immunol 9: 749, 2018. 